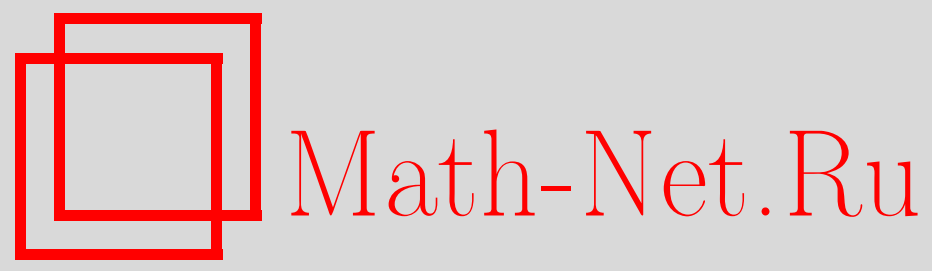

В. Ж. Сакбаев, Разрушение решений задачи Коши для нелинейного уравнений Шрёдингера, Вестн. Сам. гос. техн. ун-та. Сер. Физ.-мат. науки, 2013, выпуск 1(), 159-171

DOI: https://doi.org/10.14498/vsgtu1159

Использование Общероссийского математического портала Math-Net.Ru подразумевает, что вы прочитали и согласны с пользовательским соглашением

http: //www . mathnet.ru/rus/agreement

Параметры загрузки:

IP: 3.82 .47 .9

26 апреля 2023 г., 03:20:24

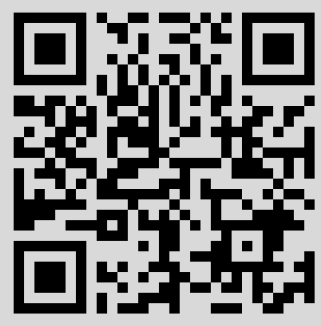


УДК 517.958:530.145.6

\section{РАЗРУШЕНИЕ РЕШЕНИЙ ЗАДАЧИ КОШИ ДЛЯ НЕЛИНЕЙНОГО УРАВНЕНИЙ ШРЁДИНГЕРА}

\section{В. Ж. Сакбаев}

Московский физико-технический институт (государственный университет), Россия, 141700, Долгопрудный, Институтский пер., 9.

E-mail: fumi2003@mail.ru

Настоящая работа посвящена изучению эфбекта конечности времени существования решения задачи Коши для нелинейного уравнения Шрёдингера. Вместе с некорректной задачей Коши рассматривается её окрестность в пространстве операторов, представляющих задачу Коши. Исследована сходимость последовательности решений задач Коши с операторами, аппроксимирующими исходньй гамильтониан.

Ключевые слова: нелинейное уравнение Шрёдингера, регуляризация, режим с обострением, разрушение решения, вязкостное решение.

Введение. Моментом разрушения решения начально-краевой задачи будем называть точную верхнюю грань правых границ промежутков существования её решения. Под явлением режима с обострением в задаче Коши понимается (см. [1-3]) существование такого решения на ограниченном временном промежутке, для которого имеет место стремление нормы решения в некотором пространстве к бесконечности на правом конце промежутка. Тем не менее разрушение решения задачи Коши может, как показывают примеры задач Коши для уравнений с частными производными первого порядка (см. [4,5]) и задач Коши с вырожденными гамильтонианами [6], не сопровождаться стремлением его нормы к бесконечности. Кроме того, уход решения на бесконечность в ряде случаев происходит в достаточно сильной норме$W_{2}^{1}\left(\mathbb{R}^{d}\right), L_{\infty}\left(\mathbb{R}^{d}\right)$, но при этом норма решения в более широком банаховом пространстве с более слабой нормой остается ограниченной равномерно на всем промежутке существования решения. Так, в случае нелинейного уравнения Шрёдингера (см. [7-9]) в момент градиентной катастрофы $T_{*}$ выполняется условие

$$
\lim _{t \rightarrow T_{*}-0}\|u(t)\|_{W_{2}^{1}\left(\mathbb{R}^{d}\right)}=+\infty,
$$

а в случае линейного (см. $[10,11])$ для последовательности $\left\{u_{n}(t)\right\}$ решений аппроксимирующих задач выполняется равенство

$$
\lim _{n \rightarrow \infty}\left\|u_{n}(t)\right\|_{W_{2}^{1}\left(\mathbb{R}^{d}\right)}=+\infty \text { при всех } t>T_{*} .
$$

Тем не менее

$$
\|u(t)\|_{L_{2}\left(\mathbb{R}^{d}\right)}=\left\|u_{0}\right\|_{L_{2}\left(\mathbb{R}^{d}\right)} \text { при всех } t \in\left[0, T_{*}\right) .
$$

В работе [12] моментом разрушения решения считается величина

$$
T_{*}=\sup \left\{t>0:\left\{x \in \mathbb{R}^{d}: \exists\left\{x_{n}\right\} \rightarrow x,\left\{t_{n}\right\} \rightarrow t-0: \lim _{n \rightarrow \infty} u\left(t_{n}, x_{n}\right)=\infty\right\}=\oslash\right\} .
$$

\footnotetext{
${ }^{1}$ Всеволод Жанович Сакбаев (к.ф.-м.н., доц.), доцент, каф. высшей математики.
} 
Поэтому возникает предположение, что задача Коши описывает эволюцию системы и на интервале времени $t>T_{*}$ в, быть может, расширенном пространстве: для уравнений Шрёдингера с градиентным обострением - в пространстве $L_{2}$ или в пространстве квантовых состояний (см. [6,13]), для уравнений с обращением решения в бесконечность на области координатного пространства - в дополнении этой области (см. [11,12,14]). Причины возникновения моментов разрушения решения дифференциальных уравнений и некоторые подходы к определению процедуры продолжения решения исследованы в работе [15].

В настоящей работе мы исследуем явление градиентного взрыва решения задачи Коши для нелинейного уравнения Шрёдингера

$$
\begin{gathered}
i \frac{d}{d t} u=\mathbf{L} u \equiv \mathbf{\Delta} u+f\left(|u|^{2}\right) u, \quad t>0, x \in \Omega \\
u(+0)=u_{0}, \quad u_{0} \in H=L_{2}\left(B_{1}\left(\mathbb{R}^{d}\right)\right) .
\end{gathered}
$$

Кроме того, исследуется поведение последовательности решений задач Коши, аппроксимирующих задачу (1), (2), решения которой допускают явление градиентного взрыва. Здесь область $\Omega$ является либо пространством $\mathbb{R}^{d}$ (в этом случае $\boldsymbol{\Delta}$ - оператор Лапласа в $\mathbb{R}^{d}$ ), либо ограниченной областью пространства $\mathbb{R}^{d}$, звездной относительно начала координат, с гладкой (задаваемой локально графиками бесконечно дифференцируемых функций) границей (в этом случае $\boldsymbol{\Delta}$ - оператор Лапласа-Дирихле в $\Omega$ ). То есть в случае отличия области $\Omega$ от пространства $\mathbb{R}^{d}$ в формулировку задачи Коши (1), (2) включается граничное условие Дирихле $\left.u\right|_{\partial \Omega}=0$.

В качестве модельного примера нелинейности в уравнении Шрёдингера будем рассматривать функцию $f\left(|u|^{2}\right)=c|u|^{p}$ с некоторой постоянной $c>0$. Помимо того будем изучать некоторый класс $\mathcal{F}$ функций $f$, определяемых нижеследующими условиями.

Условие F1. Функиия $f(s), s \in[0,+\infty)$, является $K$ раз непрерывно дифференцируемой на промежутке $[0,+\infty)$ при некотором $K \in \mathbb{N}$ (производная в точке $s=0$ понимается как одностороняя), причём $f(0)=0$.

Рассматривая комплексную плоскость $\mathbb{C}$ как двумерное вещественное линейное пространство $\mathbb{R}^{2}$, заключаем, что согласно F1 функция $f\left(|u|^{2}\right) u$ является $K$ раз непрерывно дифференцируемой как отображение из $\mathbb{R}^{2}$ в $\mathbb{R}^{2}$.

Условие F2 Существуют такие постоянные $C>0$ и $p>0$ maкие, что $\left|\frac{\partial^{|\alpha|}}{\left(\partial u_{1}\right)^{\alpha_{1}}\left(\partial u_{2}\right)^{\alpha_{2}}}\left[f\left(\left|u_{1}+i u_{2}\right|^{2}\right)\left(u_{1}+i u_{2}\right)\right]\right| \leqslant C\left(1+\left|u_{1}+i u_{2}\right|^{p}\right), \quad \forall u=u_{1}+i u_{2} \in \mathbb{C}$, при всех $\alpha=\left(\alpha_{1}, \alpha_{2}\right)$ таких, что $\alpha_{j} \in\{0,1,2, \ldots\}$ u $|\alpha|=\alpha_{1}+\alpha_{2} \leqslant K$.

В работах $[9,7,16]$ получены условия на параметр нелинейности $p$, размерность координатного пространства $d$ и начальные данные задачи Коши $(1),(2)$, достаточные для возникновения у решения задачи Коши градиентой катастрофы за конечное время, допускающее оценку сверху и, наоборот, достаточные для глобальной корректной разрешимости задачи Коши (1), (2). Близкие исследования задачи Коши с ограниченной пространственной областью $\Omega$ проведены в работе [17]. 
Рассмотрим модельный пример нелинейной функции $f\left(|u|^{2}\right)=|u|^{p}$. Причиной явления градиентной катастрофы при достаточно больших $p$ является неограниченность функционала энергии

$$
E(u)=\int_{\mathbb{R}^{d}}\left[\frac{c}{p+2}|u|^{p+2}-\frac{1}{2}|\nabla u|^{2}\right] d x,
$$

ни сверху, ни снизу. Это свойство приводит к тому, что неограниченный рост $L_{2}$-нормы градиента решения при $t \rightarrow T_{*}-0$ компенсируется неограниченным ростом при $t \rightarrow T_{*}-0$ потенциальной энергии $c\|u\|_{L_{p+2}}$ при условии постоянства энергии $E$. Поэтому в качестве регуляризующих аппроксимаций уравнения (1) рассмотрим регуляризацию нелинейного уравнения Шрёдингера четвёртого порядка, то есть последовательность нелинейных уравнений Шрёдингера

$$
i \frac{d}{d t} u=\mathbf{L}_{\varepsilon} u \equiv \Delta u+f\left(|u|^{2}\right) u+\varepsilon \Delta^{2} u, \quad t>0, \quad \varepsilon \in(0,1),
$$

где $\varepsilon \in(0,1)$ - параметр регуляризации. Тогда при $f\left(|u|^{2}\right)=|u|^{p}$ регуляризованный функционал энергии

$$
E_{\varepsilon}(u)=\int_{\mathbb{R}^{d}}\left[\frac{c}{p+2}|u|^{p+2}-\frac{1}{2}|\nabla u|^{2}+\frac{\varepsilon}{2}|\boldsymbol{\Delta} u|^{2}\right] d x, \quad \varepsilon \in(0,1),
$$

при каждом $\varepsilon \in(0,1)$ полуограничен снизу, что приводит к ограниченности поверхностей уровня энергии $E_{\varepsilon}$ в пространстве $W_{2}^{1}(\Omega)$.

1. Определение решения. Для изучения разрешимости задачи Коши введём следующие банаховы пространства.

При каждом $l \in \mathbb{N}$ через $H^{l}$ обозначим замкнутое подпространство банахова пространства $W_{2}^{l}(\Omega)$, элементы которого удовлетворяют $l$ условиям $\left.\Delta^{j} u\right|_{\partial \Omega}=0$ при $j \in \mathbb{Z}: 0 \leqslant 2 j<l$ на границе области $\Omega$.

Таким образом, $H^{2 l}=D\left(\boldsymbol{\Delta}^{l}\right)$, где $\boldsymbol{\Delta}$ - оператор Лапласа-Дирихле в области $\Omega$. Если область $\Omega$ совпадает с пространством $\mathbb{R}^{d}$, то $H^{l}=W_{2}^{l}(\Omega)$. Далее через $C(I, X)$ будем обозначать банахово пространство непрерывных отображений промежутка $I$ в банахово пространство $X$.

Следуя подходу, изложенному в $[9,16]$, дадим следующее определение.

ОПреДЕЛЕНиЕ. При каждом $l \in \mathbb{N}$ функцию

$$
u(t, \cdot) \in C\left([0, T), W_{2}^{l}(\Omega)\right) \bigcap C^{1}\left((0, T), W_{2}^{l-2}(\Omega)\right)
$$

будем называть $H_{l}$-решением задачи $(1),(2)$, если $u(t, \cdot) \in C\left([0, T), H^{l}\right)$ и выполнено равенство

$$
\lim _{t \rightarrow+0}\left\|u(t, \cdot)-u_{0}\right\|_{W_{2}^{l}(\Omega)}=0
$$

а равенство (1) при подстановке в него функции $u$ обращается в тождество при каждом $t \in(0, T)$ в пространстве $W_{2}^{l-2}(\Omega)$. 
Согласно [9] функция $u:[0, T) \rightarrow H$ является $H^{l}$-решением задачи $(1),(2)$ $(l \in\{1,2, \ldots, K\})$ тогда и только тогда, когда $u \in C\left([0, T), H^{l}\right)$ и справедливо равенство

$$
u(t)=e^{-i t \boldsymbol{\Delta}} u_{0}-i \int_{0}^{t} e^{-i(t-s) \boldsymbol{\Delta}}\left[f\left(|u(s)|^{2}\right) u(s)\right] d s, \quad t \in[0, T],
$$

$\boldsymbol{\Delta}$ - оператор Лапласа-Дирихле в области $\Omega$.

Согласно результатам работ [16] (см. предложения 2.1-2.4) и [9] (см. теорему 1.2.4 и замечание 1.2.6 цитируемой монографии) справедливы следующие утверждения.

Теорема 1. Пусть функиия $f$ удовлетворяет условиям F1 и F2 при $K \geqslant 1$. Тогда для любго $u_{0} \in H^{l}(\Omega)$ существует такое $T_{1} \in(0,+\infty]$, что задача Коши (1), (2) имеет единственное решение $u(t), t \in\left[0, T_{1}\right)$, причём если $T_{1}<+\infty$, то

$$
\lim _{t \rightarrow T_{l}-0}\|u(t)\|_{H^{1}}=+\infty
$$

Теорема 2. Пусть функиия $f$ удовлетворяет условиям F1 и F2 при некотором $K>1+d / 2$. Тогда для любых $l \in \mathbb{N}: d / 2<l \leqslant K-1$ из условия $u_{0} \in H^{l}(\Omega)$ следует существование такого числа $T_{l} \in(0,+\infty]$, что $H_{l}$-решение $u\left(t, u_{0}\right)$ задачи Коши $(1),(2)$ существует на промежутке $\left[0, T_{l}\right)$, причём если $T_{l}<+\infty$, то

$$
\lim _{t \rightarrow T_{l}-0}\left\|u\left(t, u_{0}\right)\right\|_{H^{l}}=+\infty
$$

Доказательство локального существования решения проводится на основании принципа сжимающих отображений для отображения $G$, преобразующего банахово пространство $C\left([0, T], H^{l}\right)$ при некоторых $T>0$ и $l \geqslant 1$. А именно, при каждом $l \in \mathbb{N}$ и для каждого $u_{0} \in H^{l}$ определим оператор $G$ на выпуклом замкнутом в пространстве $C\left([0, T], H^{l}\right)$ множестве

$$
M_{T}=\left\{u \in C\left([0, T], H^{l}\right), u(0)=u_{0} ;\|u\|_{C\left([0, T], H_{l}\right)} \leqslant 2\left\|u_{0}\right\|_{H^{l}}\right\}
$$

согласно равенству

$$
(G u)(t)=e^{-i t \boldsymbol{\Delta}} u_{0}-i \int_{0}^{t} e^{-i(t-s) \Delta}\left[f\left(|u(s)|^{2}\right) u(s)\right] d s, \quad t \in[0, T] .
$$

Доказательство существования и единственности неподвижной точки у отображения $G$ почти дословно повторяет доказательство теоремы 1.2 .4 работы [9].

Как и в работе [7] (см. также [18]), доказывается следующее утверждение.

Лемма 1. Если и- ${ }^{l}$-решение задачи Коши (1), (2), $l \geqslant 1$, то при значениях функиионалов $N(u)=\|u\|_{L_{2}(\Omega)}^{2}$ и энергии

$$
E(u)=\int_{\Omega}\left(\frac{1}{2} F\left(|u|^{2}\right)-\frac{1}{2}|\nabla u|^{2}\right) d x
$$


¿əe

$$
F(s)=\int_{0}^{s} f(\xi) d \xi, \quad s \geqslant 0,
$$

на функииях $u(t), t \in[0, T]$, не зависят от $t$ :

$$
N(u(t))=N\left(u_{0}\right), E(u(t))=E\left(u_{0}\right) \quad \forall t \in[0, T]
$$

ЛЕмма 2. Если функиия $F$ удовлетворяет неравенству

$$
F(s) \leqslant \frac{2 C_{0}}{p+2} s^{\frac{p+2}{2}}
$$

при некотором $C_{0}>0$ и некотором $p \in[0,4 / d)$, то $H_{1}$-решение задачи Коши $(1),(2)$ существует глобально на промежутке $[0,+\infty)$ и на указанном промежутке единственно, причём функиия $\|u(t)\|_{H_{1}}, t \in[0,+\infty)$, ограничена на промежутке $[0,+\infty)$.

Доказательств о. Согласно неравенству Гёльдера

$$
\|u\|_{p+2} \leqslant\|u\|_{2}^{1-\theta}\|u\|_{r}^{\theta}
$$

где $\theta \in(0,1), r>2$ и

$$
\frac{1}{p+2}=\frac{1-\theta}{2}+\frac{\theta}{r}
$$

и, следовательно

$$
\theta=\theta(r)=\frac{r p}{2(p+2)(r-2)} .
$$

При $d \geqslant 3$ положим

$$
r=2^{*}=\frac{2 d}{d-2}
$$

тогда

$$
\theta=d \frac{p}{2(p+2)}
$$

и согласно неравенству Соболева $\|u\|_{2^{*}} \leqslant C\|\nabla u\|_{2}$ (см. [19]). Поэтому

$$
\|u\|_{p+2} \leqslant C\|u\|_{2}^{1-\theta}\|\nabla u\|_{2}^{\theta}
$$

а поскольку $\|u\|_{2}=\left\|u_{0}\right\|_{2}$, то

$$
\|u\|_{p+2}^{p+2} \leqslant C_{1}\|\nabla u\|_{2}^{\frac{d p}{2}}
$$

Поэтому из условия сохранения энергии

$$
\frac{1}{2}\|\nabla u\|_{2}^{2}=-E\left(u_{0}\right)+\frac{1}{2} \int_{\Omega} F\left(|u|^{2}\right) d x .
$$

Тогда согласно предположению леммы

$$
\frac{1}{2}\|\nabla u\|_{2}^{2} \leqslant-E\left(u_{0}\right)+\frac{C_{0}}{p+2}\|u\|_{p+2}^{p+2} \leqslant-E\left(u_{0}\right)+C_{1}\|\nabla u\|_{2}^{\beta} .
$$


При $d \in\{1,2\} 2^{*}=+\infty$, поэтому выберем $r>2$ в (5) таким образом, чтобы выполнилось неравенство $\theta(r)(p+2)<2$, что всегда можно сделать при $p<4$ (согласно предположению леммы $p<4 / d \leqslant 4$ ). Тогда

$$
\|u\|_{p+2}^{p+2} \leqslant\|u\|_{2}^{(p+2)(1-\theta(r))}\|u\|_{r}^{(p+2) \theta(r)}
$$

в силу (5), а поскольку в силу теоремы Соболева-Гальярдо-Ниренберга при любом $r<2^{*}$ справедлива оценка $\|u\|_{r} \leqslant C_{r}\|\nabla u\|_{2}$, из условия сохранения энергии также получаем (6).

Следовательно, при всех $d \in \mathbb{N}$ справедливо неравенство

$$
\|\nabla u\|_{2}^{2}=c_{2}+c_{3}\|\nabla u\|_{2}^{\beta}
$$

где $\beta<2$. Поэтому существует такая постоянная $M>0$, что

$$
\|u(t)\|_{H_{1}}=\|\nabla u(t)\|_{2} \leqslant M \text { для любого } t \in\left[0, T_{1}\right) .
$$

Следовательно, согласно теореме 1 выполняется равенство $T_{1}=+\infty$.

Предположим для функции $f(s), s \geqslant 0$, выполнение следующего условия.

Условие F3. Функиия

$$
F(s)=\int_{0}^{s} f(\xi) d \xi
$$

удовлетворяет условию $s f(s) \geqslant C_{d} F(s) \forall s \geqslant 0$, где $C_{d}>1+2 / d$.

Например, если $f(s)=s^{p / 2}$, то условие F3 означает, что $p>4 / d$.

Теорема 3. Пусть выполнены условия теоремы 1 и условие F3. Пусть $u_{0} \in H^{1}$ и выполняются неравенства $E\left(u_{0}\right)>0 u J\left(u_{0}\right)>0$. Тогда время $T_{1}$ существования $H^{1}$-решения конечно и

$$
\lim _{t \rightarrow T_{1}-0}\|u(t, \cdot)\|_{W_{2}^{1}(\Omega)}=+\infty .
$$

Доказательство теоремы 3 повторяет рассуждения работ [7,9] об эволюции значений на $H_{1}$-решении $u(t), t \in\left(0, T_{1}\right)$ задачи $(1),(2)$ следующих функционалов:

$$
Y(u)=\int_{\Omega}|x|^{2}|u(x)|^{2} d x, \quad J(u)=\operatorname{Im} \int_{\Omega}(x, \nabla u) \bar{u} d x=\operatorname{Im} \int_{\Omega} r u_{r} \bar{u} d x,
$$

где $r=|x|$, с помощью которых на основании предположений теоремы устанавливаются соотношения

$$
\frac{d}{d t} Y(u(t))=-4 J(u(t)), \quad \frac{d}{d t} J(u(t)) \geqslant \omega\|\nabla u\|_{2}^{2},
$$

где $\omega=\omega\left(C_{d}, d\right)>0$. При получении последнего неравенства в случае ограниченной области $\Omega$ используется предположение её звёздности относительно начала координат.

Заметим, что функции $u_{0} \in C_{0}^{\infty}(\Omega)$, удовлетворяющие условиям теоремы 3, существуют.

Например, если $w \in C_{0}^{\infty}, w \neq 0$, то точки луча $\{a w, a \in(0,+\infty)\}$ удовлетворяют указанным условиям при всех достаточно больших $a$. 
2. О решении регуляризованных задач. При каждом $\varepsilon>0$ и каждом $l=$ $=0,1,2, \ldots$ будем называть $H_{l}$-решением регуляризованной задачи Коши $(2)$, (3) на промежутке $[0, T)$ функцию $u_{\varepsilon}(t, x),(t, x) \in[0, T) \times \Omega$, такую, что $u_{\varepsilon} \in C\left([0, T), H^{l}(\Omega)\right)$, удовлетворяющую равенству

$$
u_{\varepsilon}(t)=e^{-i\left(\boldsymbol{\Delta}+\varepsilon \boldsymbol{\Delta}^{2}\right) t} u_{0}+\int_{0}^{t} e^{-i\left(\boldsymbol{\Delta}+\varepsilon \boldsymbol{\Delta}^{2}\right)(t-s)} f\left(\left|u_{\varepsilon}(s)\right|^{2}\right) u_{\varepsilon}(s) d s
$$

в пространстве $C\left([0, T), H^{l}(\Omega)\right)$.

Установим условия, при которых нелинейный оператор $u \rightarrow f\left(|u|^{2}\right) u$ действует с области определения в соответствующее постановке задачи функциональное пространство. Заметим, что согласно теореме вложения С. Л. Соболева имеет место непрерывное вложение пространства $W_{2}^{l}(\Omega)$ в пространство $C(\Omega)$ при условии, что $l>d / 2$. В связи с этим нелинейный потенциал $V(u)=$ $=|u|^{p}$ является непрерывной ограниченной функцией на $\Omega$ при условии, что $u \in W_{2}^{l}(\Omega)$, где $l>d / 2$. А тогда и слагаемое $V(u) u=|u|^{p} u$ принадлежит пространству $L_{2}(\Omega)$. Те же выводы можно сделать и для нелинейной функции $f$, удовлетворяющей условиям $\mathrm{F} 1$ и $\mathrm{F} 2$, поскольку композиция $f \circ u$ является непрерывной ограниченной функцией на $\Omega$.

Утверждения следующей леммы, подобное утверждениям леммы 1.2 об уравнении Шрёдингера [18, стр. 231], доказываются с помощью применения теоремы вложения Соболева и неравенства Гёдьдера.

Лемма 3. Пусть $2(l-1) \leqslant d<2 l$ и функиия $f$ удовлетворяет условиям F1 и F2 при $K=l+1$. Тогда справедливы следующие утвержсдения:

1) для любой функиии $u \in H^{l}$ справедливь включение $f\left(|u|^{2}\right) u \in H_{l} u$ оченка $\left\|f\left(|u|^{2}\right) u\right\|_{H_{l}} \leqslant \Gamma_{l}\left(\|u\|_{H_{l}}\right)$;

2) для любых двух функиий $u, v \in H^{l}$ справедлива оценка

$$
\left\|f\left(|u|^{2}\right) u-f\left(|v|^{2}\right) v\right\|_{H_{l}} \leqslant \Gamma_{l}\left(\|u\|_{H^{l}}+\|v\|_{H^{l}}\right)\|u-v\|_{H^{l}},
$$

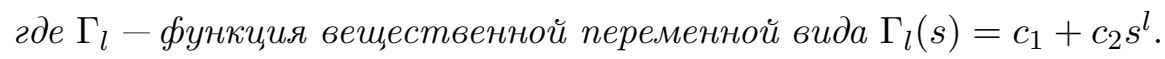

ЗАмЕчание 1. Если область $\Omega$ ограничена, то условие $f\left(|u|^{2}\right) u \in H^{l}$ включает, в частности, обращение в нуль следов $\Delta^{k} f\left(|u|^{2}\right) u$ при $2 k<l$. Это условие следует из того обстоятельства, что функция $\Delta^{k} f\left(|u|^{2}\right) u$ разлагается в сумму слагаемых, каждое из которых содержит в качестве сомножителя выражение $\Delta^{j} u, j \in 0,1, \ldots, k ;$ поэтому след $\left.\Delta^{k} f\left(|u|^{2}\right) u\right|_{\partial \Omega}=0$, если только $2 k<l$.

Теорема 4. Пусть $\varepsilon>0, K>2 u d<4 ;$ nусть $F(s) \geqslant 0$ при любом $s \geqslant 0$. Тогда для любого $u_{0} \in \dot{W}_{2}^{2}(\Omega)$ задача Коши $(2),(3)$ на промежутке $[0,+\infty)$ имеет единственное $\mathrm{H}_{2}$-решение $u_{\varepsilon}\left(t, u_{0}\right)$, причём функииональ $N(u)$ u

$$
E_{\varepsilon}(u)=\frac{1}{2} \int_{\Omega}\left(F\left(|u|^{2}-|\nabla u|^{2}+\varepsilon|\Delta u|^{2}\right) d x\right.
$$

принимаят на значениях решения $u_{\varepsilon}\left(t, u_{0}\right), t \geqslant 0$, постоянные значения.

Д о каз а тель ст в о локального существования $H_{2}$-решения задачи Коши $(2),(3)$ проводится тем же методом, что и доказательство существования локального решения задачи (1), (2) на основании принципа сжимающих 
отображений, коммутативности оператора дифференцирования и операторов полугруппы $e^{-i\left(\boldsymbol{\Delta}+\varepsilon \boldsymbol{\Delta}^{2}\right)(t)}, t>0$, и на основании оценки $\|u\|_{C(\Omega)}$ по неравенству Соболева (см. [9]). Следовательно, для всякого числа $M>0$ существует такое число $T=T(M)>0$, что для любого $u_{0} \in H^{2}(\Omega)$, удовлетворяющего условию $\left\|u_{0}\right\|_{H^{2}} \leqslant M$, задача Коши имеет решение на отрезке $[0, T(M)]$.

Так же, как и в доказательстве леммы 1, устанавливается, что на промежутке существования $H_{2}$-решения задачи Коши $(2),(3)$ величины $N\left(u_{\varepsilon}\left(t ; u_{0}\right)\right)$ и $E_{\varepsilon}\left(u_{\varepsilon}\left(t ; u_{0}\right)\right)$ сохраняют постоянные значения.

При каждом $\varepsilon \in(0,1)$ справедливо равенство

$$
E_{\varepsilon}(u)=\int_{\Omega} \frac{\varepsilon}{4}|\Delta u|^{2} d x+\int_{\Omega}\left[\frac{1}{2} F\left(|u|^{2}\right)-\frac{1}{4} \varepsilon^{-2}|u|^{2}+\frac{1}{4}\left|\varepsilon^{-1} u+\varepsilon \Delta u\right|^{2}\right] d x,
$$

согласно которому

$$
E_{\varepsilon}(u) \geqslant \int_{\Omega} \frac{\varepsilon}{4}|\Delta u|^{2} d x-\frac{1}{4} \varepsilon^{-2}\|u\|_{H}^{2}
$$

Поэтому для $H_{2}$-решения $u_{\varepsilon}\left(t ; u_{0}\right)$ справедлива оценка

$$
\int_{\Omega}\left|\Delta u_{\varepsilon}\right|^{2} d x \leqslant M_{\varepsilon}=\frac{4}{\varepsilon}\left(E_{\varepsilon}\left(u_{0}\right)+\frac{1}{4} \varepsilon^{-2}\|u\|_{H}^{2}\right)
$$

и, следовательно, $H_{2}$-норма решения ограничена на всём промежутке существования. Поэтому величина промежутка, на который может быть продолжено $H_{2}$-решение, ограничена снизу и, следовательно, $H_{2}$-решение существует на промежутке $[0,+\infty)$.

Единственность $H_{2}$-решения может быть установлена на основании липшицевости оператора $u \rightarrow f\left(|u|^{2}\right) u$ (см. лемму 3) оценки, условий F1 и F2 о нелинейной функции $f$, теоремы Соболева и леммы Гронуолла.

3. Замечание о пространствах большей размерости. Для исследования задачи $(2),(3)$ в больших размерностях $(d \geqslant 4)$ требуется ограниченность $L_{2}$ нормы производных решения порядка $d / 2$ или $(d+1) / 2$ для непрерывного вложения пространства $H^{l}(\Omega)$ в пространство $C(\bar{\Omega})$, которое, в свою очередь, необходимо для исследования задаваемого уравнением (7) отображения $G_{\varepsilon}$. Для поставленной цели следует рассмотреть регуляризации функционала энергии $E(u)$ аппроксимирующими функционалами $E_{\varepsilon, m}(u)$ более высокого порядка

$$
E_{\varepsilon, m}(u)=\int_{\mathbb{R}^{d}}\left[\frac{c}{p+2}|u|^{p+2}-\frac{1}{2}|\nabla u|^{2}+\frac{\varepsilon^{2}}{2}\left|\boldsymbol{\Delta}^{m} u\right|^{2}\right] d x, \quad \varepsilon \in(0,1), m \in \mathbb{N} .
$$

Тогда сохранение энергии $E_{\varepsilon, m}$ обеспечивает равномерную ограниченность соболевской нормы $H^{2 m}(\Omega)$, что обеспечивает равномерную ограниченность на всем промежутке существования $C(\Omega)$-нормы решения в силу непрерывности вложения пространства $W_{2}^{2 m}(\Omega)$ в пространство $C(\Omega)$ при $d<4 m$. Схема доказательства глобального существования $H^{2 m}$-решения повторяет схему доказательства теоремы 4. 
4. О сходимости последовательности решений регуляризованных задач. Далее будет исследована задача Коши с начальным условием $u_{0} \in C_{0}^{\infty}(\Omega)$, для которого время $T_{1}^{*}$ существования $H^{1}$-решение задачи Коши (1), (2) конечно в силу теоремы $3, H^{4}$-решение $u$ задачи Коши $(1)$, (2) существует на промежутке $\left[0, T_{4}^{*}\right)$ в силу теоремы 2 , а $H^{4}$-решение $u_{\varepsilon}$ любой из задач Коши $(2),(3)$ определено и равномерно ограничено на полуоси $[0,+\infty)$ в силу теоремы 4 .

ЗАмечание 2 . Очевидно, что $T_{4}^{*} \leqslant T_{1}^{*}$. Согласно работе [9], теорема 1.2.4, в случае 1 -мерной координатной области $d=1$ справедливы равенства $T_{1}^{*}=$ $=T_{2}^{*}=T_{3}^{*}=\ldots$ Действительно, в силу равенства (4), в силу неравенства Соболева, которое при $d=1$ принимает вид

$$
\|u\|_{L_{\infty}} \leqslant C\|u\|_{H^{1}} \forall u \in H^{1}
$$

и в силу условий F1 и F2 для $H^{k+1}$-решения задачи Коши $(1),(2)$ и для любого $t \in\left[0, T_{k+1}^{*}\right) \subset\left[0, T_{k}^{*}\right)$ справедлива оценка

$$
\|u(t)\|_{H^{k+1}} \leqslant C_{1}\left(\|u\|_{C\left([0, t], H^{k}\right)}\right)+C_{2}\left(\|u\|_{C\left([0, t], H^{k}\right)}\right) \int_{0}^{t}\|u(s)\|_{H^{k+1}} d s
$$

из которой следует ограниченность $H^{k+1}$-нормы решения на любом отрезке из промежутка $\left[0, T_{k}^{*}\right)$. Таким образом, если $u_{0} \in C_{0}^{\infty}(\Omega)$ и $d=1$, то $T_{k}^{*}=$ $=T_{1}^{*} \forall k \leqslant K$, но при $d>1$ имеют место неравенства $T_{1}^{*} \geqslant T_{2}^{*} \geqslant \ldots \geqslant T_{K}^{*}$.

Теорема 5. Пусть выполнены условия F1-F3, $d<4$ и $K>1+d / 2$. Пусть $u_{0} \in C_{0}^{\infty}(\Omega)$ и $T_{l}^{*} \in(0,+\infty)$ - точная верхняя грань длин промежутков, на которых существует $H^{l}$-решение задачи Коши (1), (2), $l \in \mathbb{N}$. Тогда для любого $T \in\left(0, T_{4}^{*}\right)$ справедливо равенство

$$
\lim _{\varepsilon \rightarrow 0}\left\|u(t)-u_{\varepsilon}(t)\right\|_{L_{2}(\Omega)}=0
$$

Если $T>T_{1}^{*}$, то не существует бесконечно малой последовательности $\left\{e_{k}\right\}$ такой, чтобь последовательность $\left\{u_{\varepsilon_{k}}\right\}$ сходиласъ в пространстве $L_{2}(\Omega)$ равномерно на отрезке $[0, T]$.

Доказ ат ельств в. Пусть $u_{0} \in C_{0}^{\infty}(\Omega), u(t, \cdot), t \in\left(0, T_{*}\right),-H^{4}$-решение задачи Коши (1), (2) с начальным условием $u_{0}$ и градиентной катастрофой при $t \rightarrow T_{*}-0$, пусть $T \in\left(0, T_{*}\right)$ и $u_{\varepsilon}(t)$ - решение задачи Коши $(2),(3)$ при некотором $\varepsilon>0$ с начальным условием $u_{0}$.

Докажем, что

$$
\lim _{\varepsilon \rightarrow 0} \sup _{t \in[0, T]}\left\|u_{\varepsilon}(t)-u(t)\right\|_{L_{2}(\Omega)}=0
$$

Функция $v_{\varepsilon}(t)=u_{\varepsilon}(t)-u(t), t \in[0, T]$, удовлетворяет начальному условию $v_{\varepsilon}(+0)=0$ и принимает значения в пространстве $H^{4}$. Следовательно, функция $v_{\varepsilon}$ Удовлетворяет дифференциальному уравнению 


$$
\begin{aligned}
i \frac{\partial}{\partial t} v_{\varepsilon}(t, x)=[\Delta+ & \left.\varepsilon \Delta^{2}+f\left(|u(t, x)|^{2}\right)\right] v_{\varepsilon}(t, x)+ \\
+\left[f\left(\left|u_{\varepsilon}(t, x)\right|^{2}\right)-\right. & \left.f(|u(t, x)|)^{2}\right] u_{\varepsilon}(t, x)+ \\
& +\varepsilon \Delta^{2} u(t, x), \quad(t, x) \in(0, T) \times \Omega .
\end{aligned}
$$

Поскольку $K>1$, в силу теоремы Лагранжа для любых $s_{1}, s_{2} \in \mathbb{R}_{+}$существует такое число $\theta\left(s_{1}, s_{2}\right) \in(0,1)$, что

$$
f\left(s_{1}\right)-f\left(s_{2}\right)=f^{\prime}\left(s_{1}+\theta\left(s_{1}-s_{2}\right)\right)\left(s_{1}-s_{2}\right),
$$

следовательно,

$$
\begin{aligned}
& f\left(\left|u_{\varepsilon}(t, x)\right|^{2}\right)-f\left(|u(t, x)|^{2}\right)= \\
& \quad=f^{\prime}\left(|u(t, x)|^{2}+\theta(t, x)\left(\left|u_{\varepsilon}(t, x)\right|^{2}-|u(t, x)|^{2}\right)\right)\left(\left|u_{\varepsilon}(t, x)\right|^{2}-|u(t, x)|^{2}\right) .
\end{aligned}
$$

При этом $f\left(\left|u_{\varepsilon}\right|^{2}\right)-f\left(|u|^{2}\right), v_{\varepsilon} \in C([0, T] \times \bar{\Omega})$, поскольку $u_{\varepsilon}, u \in C([0, T] \times \bar{\Omega})$. Поэтому функция

$$
\begin{aligned}
& Q_{\varepsilon}(t, x) \equiv f^{\prime}\left(|u(t, x)|^{2}+\theta(t, x)\left(\left|u_{\varepsilon}(t, x)\right|^{2}-|u(t, x)|^{2}\right)\right)= \\
& =\left(f\left(\left|u_{\varepsilon}(t, x)\right|^{2}\right)-f\left(|u(t, x)|^{2}\right)\right)\left(\left|u_{\varepsilon}(t, x)\right|^{2}-|u(t, x)|^{2}\right)^{-1}, \quad(t, x) \in[0, T] \times \bar{\Omega}
\end{aligned}
$$

является непрерывной, ибо на открытом множестве

$$
D_{+}=\{(t, x) \in(0, T) \times \Omega:|v(t, x)|>0\}
$$

является композицией непрерывных функций, а на его границе имеет предельное значение $f^{\prime}\left(|u(t, x)|^{2}\right)$. Следовательно, функция $Q_{\varepsilon}(t, x),(t, x) \in D_{+}$, доопределённая на множестве $[0, T] \times \bar{\Omega} \backslash D_{+}$равенством

$$
Q_{\varepsilon}(t, x)=f^{\prime}\left(|u(t, x)|^{2}\right),
$$

непрерывна на множестве $[0, T] \times \bar{\Omega}$. Следовательно, $Q_{\varepsilon} \in C([0, T] \times \bar{\Omega})$ при каждом значении параметра $\varepsilon$, так как $f^{\prime}(s) \in C([0,+\infty))$.

Далее, для любых $(t, x) \in[0, T] \times \bar{\Omega}$ справедливо равенство

$$
\left|u_{\varepsilon}(t, x)\right|^{2}-|u(t, x)|^{2}=v_{\varepsilon}(t, x) \bar{u}_{\varepsilon}(t, x)+u(t, x) \bar{v}_{\varepsilon}(t, x) .
$$

Поэтому для каждого $(t, x) \in[0, T] \times \bar{\Omega}$ выполняется равенство

$$
\left[f\left(\left|u_{\varepsilon}(t, x)\right|^{2}\right)-f\left(|u(t, x)|^{2}\right)\right] u_{\varepsilon}(t, x)=K_{\varepsilon}(t, x) v_{\varepsilon}(t, x)+J_{\varepsilon}(t, x) \bar{v}_{\varepsilon}(t, x),
$$

где функции

$K_{\varepsilon}(t, x)=Q_{\varepsilon}(t, x)\left|u_{\varepsilon}(t, x)\right|^{2}, \quad J_{\varepsilon}(t, x)=Q_{\varepsilon}(t, x) u(t, x) u_{\varepsilon}(t, x),(t, x) \in[0, T] \times \bar{\Omega}$

при каждом значении параметра $\varepsilon \in(0,1)$ лежат в пространстве $C([0, T] \times \bar{\Omega})$.

Определим функцию $w_{\varepsilon}(t)=\left(v_{\varepsilon}(t), \bar{v}_{\varepsilon}(t)\right)^{\top}, t \in[0, T]$, со значениями в пространстве $\mathcal{H}=H \oplus H$. Тогда согласно (8) вектор-функция $w_{\varepsilon}(t), t \in$ 
$[0, T]$, является слабым (в смысле интегрального тождества) решением задачи Коши для линейного уравнения Шрёдингера

$$
i \frac{d}{d t} w_{\varepsilon}(t)-\mathcal{L}_{\varepsilon}(t) w_{\varepsilon}(t)=\mathcal{F}_{\varepsilon}(t)
$$

с нулевым начальным условием $w_{\varepsilon}(0)=(0,0)^{\top}$. Здесь

$$
\begin{gathered}
\mathcal{F}_{\varepsilon}(t)=\varepsilon \Delta^{2} u(t), \quad t \in[0, T], \\
\mathcal{L}_{\varepsilon}(t) w_{\varepsilon}(t)=\left(\begin{array}{cc}
\boldsymbol{\Lambda}_{\varepsilon}(t) & 0 \\
0 & \boldsymbol{\Lambda}_{\varepsilon}(t)
\end{array}\right) w_{\varepsilon}(t)+ \\
\\
\quad+\left(\begin{array}{cc}
\mathbf{K}_{\varepsilon}(t) & 0 \\
0 & \mathbf{K}_{\varepsilon}(t)
\end{array}\right) w_{\varepsilon}(t)+\left(\begin{array}{cc}
0 & \mathbf{J}_{\varepsilon}(t) \\
\overline{\mathbf{J}}_{\varepsilon}(t) & 0
\end{array}\right) w_{\varepsilon}(t),
\end{gathered}
$$

оператор $\boldsymbol{\Lambda}_{\varepsilon}(t)$ действует на функцию $v_{\varepsilon}(t)$ согласно правилу

$$
\boldsymbol{\Lambda}_{\varepsilon}(t) v_{\varepsilon}(t)=\left[\Delta+\varepsilon \Delta^{2}+f\left(|u(t)|^{2}\right)\right] v_{\varepsilon}(t),
$$
а оператор-функции
согласно правилам $\left(\begin{array}{cc}\mathbf{K}_{\varepsilon}(t) & 0 \\ 0 & \mathbf{K}_{\varepsilon}(t)\end{array}\right)$ и $\left(\begin{array}{cc}0 & \mathbf{J}_{\varepsilon}(t) \\ \overline{\mathbf{J}}_{\varepsilon}(t) & 0\end{array}\right)$ действуют на $w_{\varepsilon}(t)$

$$
\begin{gathered}
\left(\begin{array}{cc}
\mathbf{K}_{\varepsilon}(t) & 0 \\
0 & \mathbf{K}_{\varepsilon}(t)
\end{array}\right)\left(\begin{array}{l}
v_{\varepsilon}(t) \\
\bar{v}_{\varepsilon}(t)
\end{array}\right)=\left(\begin{array}{c}
K_{\varepsilon}(t, x) v_{\varepsilon}(t) \\
K_{\varepsilon}(t, x) \bar{v}_{\varepsilon}(t)
\end{array}\right) \\
\left(\begin{array}{cc}
0 & \mathbf{J}_{\varepsilon}(t) \\
\overline{\mathbf{J}}_{\varepsilon}(t) & 0
\end{array}\right)\left(\begin{array}{l}
v_{\varepsilon}(t) \\
\bar{v}_{\varepsilon}(t)
\end{array}\right)=\left(\begin{array}{l}
J_{\varepsilon}(t, x) \bar{v}_{\varepsilon}(t) \\
\bar{J}_{\varepsilon}(t, x) v_{\varepsilon}(t)
\end{array}\right) .
\end{gathered}
$$

Как установлено выше, при каждом $\varepsilon \in(0,1)$ функции $K_{\varepsilon}, J_{\varepsilon}$ непрерывны на множестве $[0, T] \times \Omega$, поэтому при каждом $\varepsilon \in(0,1)$ операторы $\mathcal{L}_{\varepsilon}(t)$, $t \in[0, T]$, имеют не зависящую от параметров $(t, \varepsilon) \in[0, T] \times(0,1)$ область определения $D\left(\mathcal{L}_{\varepsilon}(t)\right)=H^{4} \oplus H^{4}$, являются самосопряжёнными операторами в пространстве $\mathcal{H}$ при всех $(t, \varepsilon) \in[0, T] \times(0,1)$, а при фиксированном $\varepsilon \in(0,1)$ нестационарная часть оператора $\mathcal{L}_{\varepsilon}$ зависит от параметра $t \in[0, T]$ непрерывно в сильной операторной топологии. Следовательно, задача Коши с оператором $\mathcal{L}_{\varepsilon}(t)$ является равномерно корректной (см. [20, п. 2.3]). Однородная задача Коши для уравнения Шрёдингера с зависящим от времени гамильтонианом $\mathcal{L}_{\varepsilon}(t)$ порождает унитарное двухпараметрическое эволюционное семейство $\mathcal{W}_{\varepsilon}(t, s), 0 \leqslant s \leqslant t<+\infty$, которое позволит определить решение неоднородной задачи по формуле

$$
v_{\varepsilon}(t)=\varepsilon \int_{0}^{t} \mathcal{W}_{\varepsilon}(t, s) \Delta u(s) d s, \quad t \in[0, T]
$$

Поскольку операторы $\mathcal{W}_{\varepsilon}(t, s)$ унитарны, в силу оценки $u \in C\left([0, T], W_{2}^{2}(\Omega)\right)$ заключаем, что

$$
\lim _{\varepsilon \rightarrow 0} \sup _{t \in[0, T]}\left\|u_{\varepsilon}(t)-u(t)\right\|_{H}=0
$$


что и доказывает утверждение теоремы о сходимости.

Отсутствие предела решения $u(t), t \in\left[0, T_{*}\right)$, в пространстве $H$ по произвольной последовательности $\left\{t_{k}\right\}$ такой, что $t_{k} \rightarrow T_{*}-0$ при $k \rightarrow \infty$, установленное в работе [8], противоречит сходимости последовательности $\left\{u_{\varepsilon}(t)\right.$, $\left.t \in\left[0, T_{*}\right]\right\}$ при $\varepsilon \rightarrow 0$ в пространстве $C\left(\left[0, T_{*}\right], H\right)$ и установленной выше сходимости последовательности $\left\{u_{\varepsilon}(t), t \in[0, \tau]\right\}$ при $\varepsilon \rightarrow 0$ в пространстве $C([0, \tau], H)$ при произвольном $\tau>0$.

ЗАмечание 3. Установленное в теореме 5 отсутствие сходящихся в пространстве $C([0, T], H)$ последовательностей регуляризованных решений характерно для аппроксимаций глобально вырождающихся операторов Шрёдингера (см. [6]). В работах $[13,15]$ предложены методы исследования предельного поведения равномерно ограниченной расходящейся в пространстве $C([0, T], H)$ последовательности $u_{\varepsilon}$ при $\varepsilon \rightarrow 0$.

\section{БИБЛИОГРАФИЧЕСКИЙ СПИСОК}

1. А. А. Самарский, В. А. Галактионов, С. П. Курдюмов, А. Г. Михайлов, Режимы с обострением в задачах для квазилинейных параболических уравнений. М.: Наука, 1987. 478 c. [A. A. Samarskiy, V. A. Galaktionov, S. P. Kurdyumov, A. P. Mikhaylov, Peaking modes in problems for quasilinear parabolic equations. Moscow: Nauka, 1987. 478 pp.]

2. Э. Митидиери, С. И. Похожаев, "Априорные оценки и отсутствие решений нелинейных уравнений и неравенств в частных производных" / Тр. МИАН, Т. 234. М.: Наука, 2001. C. 3-383; англ. пер.: E. Mitidieri, S. I. Pohozaev A priori estimates and blow-up of solutions to nonlinear partial differential equations and inequalities // Proc. Steklov Inst. Math., 2001. Vol. 234. Pp. 1-362.

3. H. Fujita, "On the blowing up of solutions of the Cauchy problem for $u_{t}=\Delta u+u^{1+\alpha}$ " // J. Fac. Sci. Univ. Tokyo Sect. I, 1966. Vol.13. Pp. 109-124.

4. С. Н. Кружков, Лекции по уравнениям с частными производными. М.: МГУ, 1970. [S. N. Kruzhkov, Lectures on partial differential equations. Moscow: Moscow State Univ., 1970].

5. О. А. Олейник, E. В. Радкевич, Уравнения с неотрицательной характеристической формой. М.: Московск. ун-т, 2010. 360 с. [O. A. Oleynik, E. V. Radkevich, Equations with nonnegative characteristic form. Moscow: Moscow Univ., 2010. 360 pp.]

6. В. Ж. Сакбаев, “Задача Коши для линейного дифференциального уравнения с вырождением и усреднение аппроксимирующих ее регуляризаций” / В сб.: Уравнения в частных производных / СМФН, Т. 43. М.: РУДН, 2012. С. 3-172. [V. Zh. Sakbaev, "Тhe Cauchy problem for a linear differential equation with degeneration and averaging of its approximating regularizations" / In: Partial differential equations / CMFD, 43. Moscow: PFUR, 2012. Pp. 3-172].

7. R. T. Glassey, "On the blowing up of solution to the Cauchy Problem for nonlinear Schrödinger equations" // J. Math. Phys, 1977. Vol. 18, no. 9. Pp. 1794-1797.

8. F. Merle, Y. Tsutsumi, " $L_{2}$ convergence of blow-up solutions for nonlinear Shrodinger equation with critical power nonlinearity" // J. Differ. Equations, 1990. Vol. 84, no. 2. Pp. 205-214.

9. P. E. Zhidkov, "Korteweg-de Vries and nonlitear Schrödinger equations: qualitative theory. Lecture Notes in Math" / Lecture Notes in Mathematics, 1756. Berlin: Springer-Verlag, 2001. vi+147 pp.

10. P. Baras, J. Goldstein, "The heat equation with a singular potential" // Trans. Amer. Math. Soc., 1984. Vol. 284, no. 1. Pp. 121-139.

11. V. A. Galaktionov, I. V. Kamotski, "On nonexistance of Baras-Goldstein type for higherorder parabolic eqations with singular potential" // Trans. Amer. Math. Soc., 2010. Vol. 362, no. 8. Pp. 4117-4136. 
12. N. Mizoguchi, F. Quirós, J. L. Vázquez, "Multiple blow-up for a porous medium equation with reaction" // Math. Ann., 2011. Vol. 350, no. 4. Pp. 801-827.

13. В. Ж. Сакбаев, "Об усреднении квантовых динамических полугрупп" // TMФ, 2010. T. 164, № 3. C. 455-463; англ. пер.: V. Zh. Sakbaev, "Averaging of quantum dynamical semigroups" // Theoret. and Math. Phys., 2010. Vol. 164, no. 3. Pp. 1215-1221.

14. V. A. Galaktionov, J. L. Vázquez, "Necessary and sufficient conditions for complete blowup and extinction for one-dimensional quasilinear heat equations" // Arch. Rational Mech. Anal., 1995. Vol. 129, no. 3. Pp. 225-244.

15. V. Zh. Sakbaev, "On the properties of ambiguity and irreversibility of dynamical maps of initial data space of Cauchy problems" // p-Adic Numbers Ultrametric Anal. Appl., 2012. Vol. 4, no. 4. Pp. 306-318.

16. J. Ginibre, G. Velo, "On a class of nonlinear Schrödinger equations. I. The Cauchy problem, general case" // J. Funct. Anal., 1979. Vol. 32, no. 1. Pp. 1-32.

17. O. Kavian, "A remark on the blowing-up solutions to the Cauchy problem for nonlinear Schrödinger equation" // Trans. Amer. Math. Soc., 1987. Vol. 299, no.1. Pp. 192-203.

18. A. Pazy, "Semigroups of linear operators and applications to partial differential equations" / Applied Mathematical Sciences, 44. New York: Springer-Verlag, 1983. viii+279 pp.

19. С. М. Николъский, Приближение функций многих переменных и теоремы вложения. M.: Наука, 1969. 480 c. [S. M. Nikol'skiy, Approximation of functions of several variables and imbedding theorems. Moscow: Nauka, 1969. 480 pp.]

20. С. Г. Крейн, Линейные дифференциальные уравнения в банаховых пространствах. М.: Наука, 1967. 464 c. [S. G. Kreyn, Linear differential equations in a Banach space. Moscow: Nauka, 1967. 464 pp.]

Поступила в редакцию 12/XI/2012;

в окончательном варианте - 01/II/2013.

MSC: $46 \mathrm{N50} ; 81 \mathrm{~S} 15,81 \mathrm{P} 15$

\section{BLOW-UP OF SOLUTIONS OF CAUCHY PROBLEM FOR NONLINEAR SCHRÖDINGER EQUATION}

\section{V.Zh. Sakbaev}

Moscow Institute of Physics and Technology (State University),

9, Inststitutskii per., Dolgoprudny, 141700, Russia.

E-mail: fumi2003@mail.ru

In this work we study the effect of time finiteness of the existence of Cauchy problem for nonlinear Schrödinger equation solution. Together with the ill-posed Cauchy problem we consider its neighborhood in the space of operators, representing Cauchy problem. We explore the convergence of sequence of solutions of Cauchy problems with the operators, approximating the initial Hamiltonian.

Key words: nonlinear Schrödinger equation, regularization, blow-up regime, blow-up of solution, viscosity solution.

Original article submitted $12 / \mathrm{XI} / 2012$; revision submitted $01 / \mathrm{II} / 2013$.

Vsevolod Zh. Sakbaev (Ph. D. (Phys. \& Math.)), Associate Professor, Dept. of Higher Mathematics. 